\title{
The Research on the Design of the Patient Information System Terminal
}

\author{
Yuchen Yang ${ }^{1,}$, , Lili Liu ${ }^{2, b^{*}}$ \\ 1 Jiangsu Key Laboratory of Large Engineering Equipment Detection and Control, Xuzhou Institute \\ of Technology, Xuzhou, Jiangsu Province, 221000,China \\ 2 Jiangsu Key Laboratory of Large Engineering Equipment Detection and Control, Xuzhou Institute \\ of Technology, Xuzhou, Jiangsu Province, 221000,China \\ ay1285501947@163.com, bdxwll@ @ina.com
}

Keywords: Patient Information. Humanization form Design. Interface Design

Abstract: this paper mainly analyzes the patient information system flow and its interface design by the behavior and demand of patients, and puts forward the design concept of the information terminal product. We had a preliminary design of the product form and we divided the system into different level in terms of its interface and then researched its humanized design.

The patient information terminal, installed beside each bed, connects with the server by wire, which is beneficial to the sickroom management and the use of patients. On the one hand, it is a communication tool which can feedback the patients' situation and information to nurses in time, tell doctors the patient's feeling during the treatment and requirements on the treatment and medication, and communicate with friends through voice and video. On the other hand, it is also a web browser which can link to the hospital internal network to inquiry the situation of illness, medication and the use of funds, and to an external network to deal with other things such as the disease-related knowledge, national affairs, urgent work; it also can be used as a kind of entertainment tools. The patient information terminal also use hardware device on the computer, and includes a variety of input and output devices in order to meet the various needs of patients[1].

\section{Analysis on the concept of the patient information terminal}

The patient information system is a terminal product through which patients can communicate with nurses and the hospital information system in the bed. Patients can move the screen to the front of themselves and adjust the position and height of it according to his need, and can open the screen through wireless device or have a direct dialogue with nurses[2,3]. By navigation keys, they can operate the screen to experience various functions such as information research, entertainment, and connection with friends.
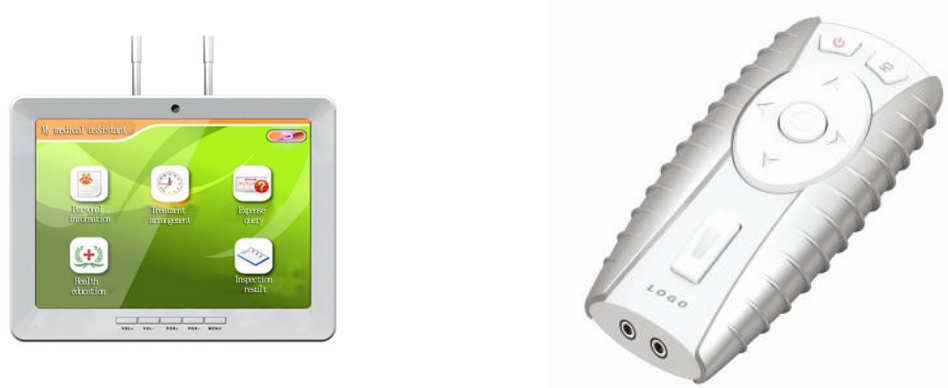

Figure 1 the Final Appearance of Patient Information Terminal 


\section{Form Design of the Patient Information Terminal}

Patient information terminal consists of two parts. One is the display which can be lifted and dropped; the other is the remote control which can control the display terminal and call. The information terminal is softly shaped. Besides, it tends to be more modern and fresh and it is still given priority to with white attune, with a little warm. Display terminal, with 12 inches (or more) screen, is pensile on the ceiling above the patients' bed [4]. Its movements are controlled by the remote control. Cameras can satisfy people's need to contact through videos. Remote control function keys consist of navigation button, switch button, function switch button and word calling button. The keys are shaped with accurate proportions and the non-slip design is beneficial for patients to grasp. When using the terminal, patients just need to press the remote control lightly to meet the requirement for information communication. So, the terminal greatly relieves the psychological stress which is caused by hospitalization and facilitates communication between doctor and patient. The main functions of the display device are embedded in the interface design [5].

\section{Interface Design of Patient Information Terminator}

The design of the patient terminal interface is mainly divided into three level interface. The key function interface have 4 kinds of functions :patient information, hospital services, leisure and entertainment and external connection.

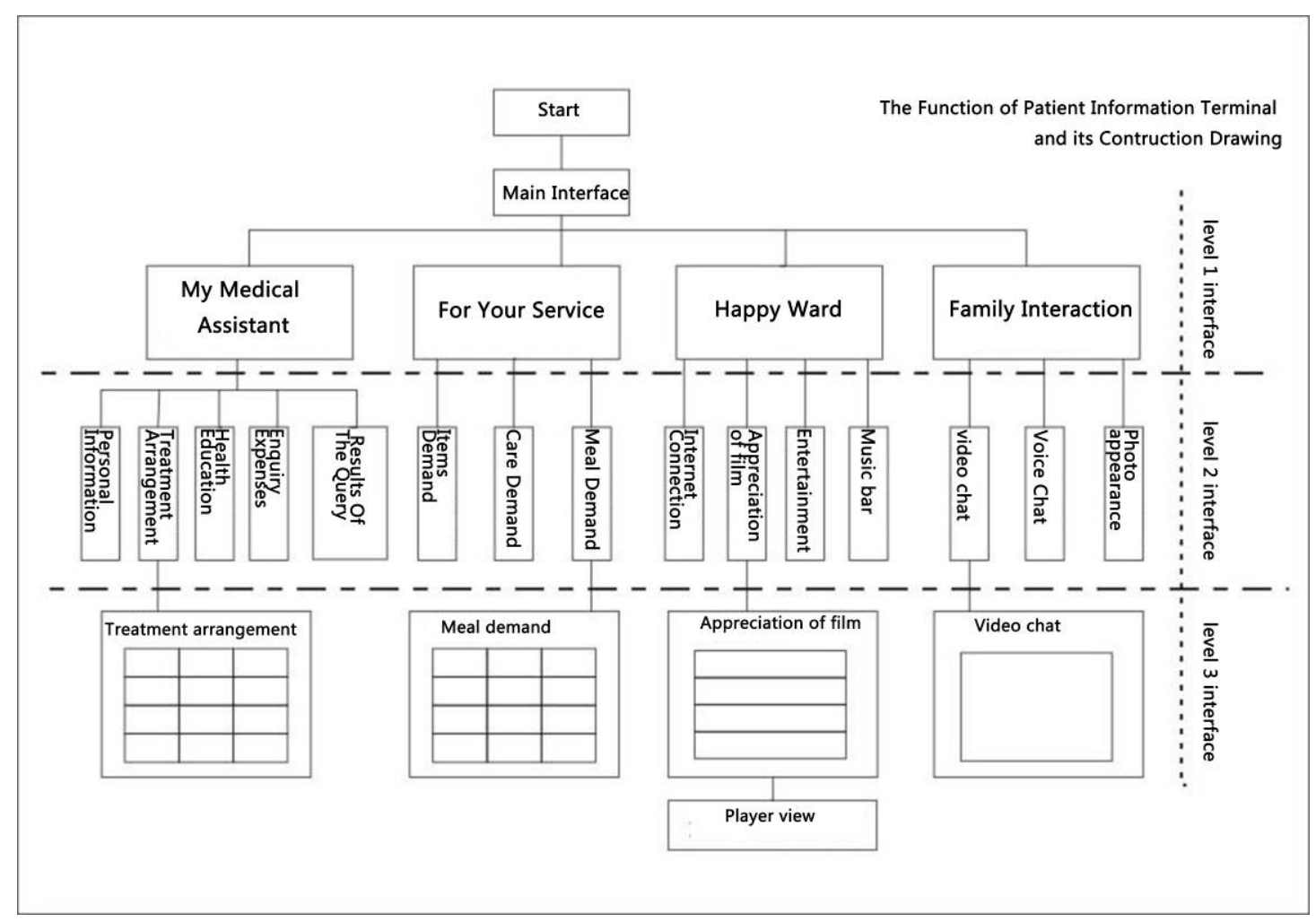

Figure 2 the Function of Patient Information Terminal and its Contraction Drawing

\section{(1)Key interface(level 1 interface)}

The key interface of the patient terminal is the main navigation interface, showing four main navigation buttons, which is grouped in terms of function: patient information, hospital services, leisure and entertainment and external relations. Considering from the patients' perspectives, it has 
a humanized name design and changes these four names to "my medical assistant", "for your service", "happy ward" and "family interaction" respectively[5]. The interface background is green, which gives a feeling of freshness and pleasure. When you click the navigation keys on the remote controller to choose and confirm, the system will enter the relevant level 2 interface.

\section{(2)Level 2 interface}

The interface of "My medical assistance" is used by patients to get medical information which is related to their own[6]. It has 5 icons: personal data; treatment arrangement; health education; expense query; inspection results query.

The interface of 'at your service' is used by patients to order related services of the hospital. It includes demand of items and care and meal service. The interface of 'happy ward' is to meet the patients' demands of entertainment. It includes the internet service, film appreciation, playing game and music appreciation est. The interface of 'interaction between families' is used by patients to communication with their relatives or friends online. It includes the functions of video chat, voice chat, taking photos and videos[7].

\section{(3)Level 3 interface}

The level 3 interface is an interface interacting with each specific function under each level 2 interface. For example, patients choose the function of 'my medical assistant'-- 'treatment arrangement' and then the screen will display this 3-level interface as shown in figure 3-patient's daily treatment programs and schedule[8].

\section{Conclusion:}

The patient information terminal's role is to optimize work process, to improve work efficiency, to reduce the labor intensity of nursing staff,to strengthen between doctor and nurse and patient communication and coordination, to improve patient satisfaction. The patient information system is the goal of the work process of executive orders into the information management, to replace the paper implementation form, to achieve paperless medical advice, to solve problems of paper orders; in the care of patients,. The patient information system will help nurses to accurately grasp the state of the patient at any time, work beside the patient, guarantee medical safety, timely solve patient problems at the same time the ward calling system together, to improve the work efficiency, spend more time taking care of a patient, the realization of safe, efficient, human-oriented medical requirements. In addition, through the information terminal, the patient will be more timely and effectively communicate with medical staff, more positive to cooperate with the treatment. It can also be used to learn about their disease with important information and health knowledge, also can make the appropriate leisure activities, learn more about the outside information and enhance interpersonal and emotional communication with family and friends, which give patients a more humanized hospital psychological environment.

\section{Reference:}

[1] Liu Xinxiong, Zhao Wei. The medical products industrial design research on [J]. machine design and research, 2005,21 (6).

[2] Yuan Hefa, Ding Wei, Chen Shen Ren. Medical equipment humanized design of [J]. machine design and research, 2004,20 (5): 81-82.

[3] Cheng Yongliang medical instrument design of UCD based on [J]. Chinese medical 
equipment information, 2005,13 (3): 30-38.

[4] Li Haiyan, Li Jiuxi. The emotional design of [J]. China medical information of medical equipment, 2006,12 (11): 19-25.

[5] Dou Jinhua, Xiao Dihu. Decorative sense of security design of [J]. medical products, 2006,3:121.

[6] Wang Chuan. Search engine user behavior model based on [J]. Computer Engineering, 2008,34 (4).

[7] [D]. Yuan Pinjun. Study on detection of medical product interface design theory and application of Chengdu: Sichuan University, 2006:28

[8] Ding Yulan, Guo Gang, Zhao Jianghong. The man-machine engineering [M]. Beijing, Beijing Institute of Technology press, 2000 